\title{
Thermoelectric Power in the Double Exchange Model
}

\author{
Beom Hyun $\mathrm{Kim}^{1}$, Unjon $\mathrm{Yu}^{2}$, Kyoo $\mathrm{Kim}^{1}$, and B. I. Min ${ }^{1 *}$ \\ ${ }^{1}$ PCTP, Department of Physics, Pohang University of Science and Technology, Pohang 790-784, Korea \\ ${ }^{2}$ Theoretical Physics III, Center for Electronic Correlations and Magnetism, \\ Institute for Physics, University of Augsburg, D-86135 Augsburg, Germany
}

(Dated: November 4, 2018)

\begin{abstract}
Employing the Monte-Carlo method and the exact diagonalization, we have investigated the temperature dependence of the thermoelectric power (TEP) for the double exchange model in the dilute carrier concentration limit. We have found that the TEP follows the Heikes formula in the high temperature regime, whereas, in the intermediate temperature regime, the TEP is suppressed by the exchange coupling between itinerant electrons and local spins. In the low temperature regime, the TEP exhibits an anomalous peak and dip feature near the magnetic transition temperature $T_{C}$ which can be understood based on the magnetic polaron state. We have also found that the TEP, in the presence of the magnetic field, shows the positive magnetothermoelectric power near $T_{C}$.
\end{abstract}

PACS numbers: 75.40.Mg, 75.47.-m, 75.40.Cx

The strong coupling between the electrical transport and the magnetism appears in the systems which show the transition from the paramagnetic $(\mathrm{PM})$ insulator to the ferromagnetic (FM) metal. Colossal magnetoresistance (CMR) doped manganese oxides 1], dilute magnetic semiconductors [2, 3], and $\mathrm{EuB}_{6}$ [4, 5] are typical examples. Simultaneous FM and metallic transitions have been well described by the double exchange model in which the itinerant electrons are coupled to the local spins through the Hund-type exchange interaction. 6, 7, 8, 9]

In these systems, the thermoelectric power (TEP) as well as the electrical conductivity shows an anomalous behavior due to a strong correlation between conduction electrons and local spins. For example, depending on the doping ratio, applied magnetic field, or temperature, doped perovskite manganese oxides exhibit intriguing TEP behaviors such as the sign change, the appearance of a large peak in the FM phase, and the magnetothermoelectric effect. [10, 11, 12, 13] The TEP would be affected by various interactions such as electron-electron, electron-phonon, Jahn-Teller interaction, etc. The essential feature of the TEP in doped perovskite manganese oxides, however, can be the explained by the double exchange mechanism.

It has been well known that the double exchange model in the dilute concentration limit produces the magnetic polaron. The magnetic polaron is a composite quasiparticle of a charge carrier and the magnetic polarization field of local magnetic moments induced by the carrier. 14, 15] The magnetic polaron effect enhances the effective mass of the carrier and can localize carriers. Thus the magnetic polaron state is featured by FM clusters embedded in the paramagnetic background and by the insulating behavior in the resistivity. 16]

In this study, we have examined the TEP behavior for

\footnotetext{
${ }^{*}$ Corresponding author: bimin@physics. postech.ac.kr
}

the double exchange model in the dilute concentration limit. By using the Kubo's formalism, we have obtained the temperature dependent TEP and investigated its behavior with respect to the exchange coupling strength. Further, we have demonstrated the field dependence of the TEP near the magnetic transition temperature $T_{C}$.

The double exchange model which includes the strong exchange interaction between itinerant electrons and local spins has the following Hamiltonian form:

$$
H=-t \sum_{\langle i, j\rangle \sigma}\left(c_{i \sigma}^{\dagger} c_{j \sigma}+\text { h.c. }\right)-J_{H} \sum_{i} \vec{\sigma}_{i} \cdot \vec{S}_{i},
$$

where $\vec{S}_{i}$ and $\vec{\sigma}_{i}$ denote the spin operators of the local and the itinerant electron at the $i$-th site, respectively. $c_{i \sigma}$ $\left(c_{i \sigma}^{\dagger}\right)$ is the annihilation (creation) operator of the electron with the $\sigma$ spin state at the $i$-th site, $t$ is the hopping parameter, and $J_{H}$ represents the exchange coupling between local spins and electrons. For simplicity, we have considered, in this study, a two-dimensional $(2 D)$ square lattice system with Ising-type local spins $\left(S_{z}= \pm \frac{1}{2}\right)$. Previous studies show that the simulation results of the double exchange model do not depend much on the dimensionality and lattice type. [17, [18] We have neglected the electron-electron interaction. The electron-electron interaction can give rise to the important effect on the electrical and thermal transport properties. To avoid its effect, we have considered a system with very low carrier concentration.

In order to calculate the TEP for the double exchange model, we have employed the Kubo's formalism of the transport coefficients for a system in the presence of both electric field and temperature gradient. 19, 20, 21] The thermoelectric power is given by

$$
S=\frac{1}{T} \frac{M_{12}}{M_{11}}+\frac{\mu}{|e| T},
$$

where $e$ is the electron charge and $\mu$ is the chemical po- 
tential. The transport coefficients $M_{i j}$ are defined by

$$
\begin{aligned}
M_{i j}= & \lim _{\omega \rightarrow 0}\left\{D_{i j} \delta(\omega)\right. \\
& \left.+\frac{1}{N \hbar} \frac{1-e^{-\beta \hbar \omega}}{2 \omega} \int_{-\infty}^{\infty} d t e^{i \omega t}\left\langle\hat{J}_{i}(t) \hat{J}_{j}(0)\right\rangle\right\}
\end{aligned}
$$

where $N$ is the size of lattice, $D_{11}$ and $D_{12}$ are the general stiffnesses of the charge and energy, and $\hat{J}_{1}$ and $\hat{J}_{2}$ represent their current operators, respectively.

Using the Monte-Carlo (MC) method combined with the exact diagonalization (ED), we have calculated the thermal average of the TEP. For the description of thermal contribution of local spins, we have used the MC method based on the standard Metropolis algorithm 23. with the periodic boundary condition. The electronic energy and states are obtained through the ED method. 24]

In the high temperature limit, the TEP for the double exchange model becomes nearly constant and simply proportional to the entropy per carrier. It is because the first term in Eq. 2 becomes zero at high temperature and only the second term contributes to the TEP. Then the TEP is expressed by the following Heikes formula: 22]

$$
S(T \rightarrow \infty) \rightarrow \frac{\mu}{|e| T}=\frac{s}{|e|}=-\frac{k_{B}}{|e|} \ln \frac{2-n}{n},
$$

where $s$ is the entropy per electron, and $n$ is the electron density. This feature is well described in Fig. 1(a). Regardless of the magnitude of $J_{H}$, all the curves of the TEP converge to the specific horizontal line. This asymptotic line in Fig. 1(a) represents the value of the TEP $\left(S=-4.8442 k_{B} /|e|\right)$ for the case of dilute concentration $n=0.0156$.

In the intermediate temperature regime $(0.5<T<$ 10.0), the TEP exhibits different behaviors depending on the strength of $J_{H}$. As shown in Fig. 1(a), the value of TEP is the largest for $J_{H}=0.0$, and becomes reduced with increasing $J_{H}$. It is because the strong exchange coupling between an itinerant electron and local spin brings down the spin entropy of conduction electrons and inhibits the heat current.

In the low temperature limit $(T<0.5)$, the TEP for the double exchange model shows interesting behavior for finite $J_{H}$. The inset in Fig. 1(a) reveals that a peak and dip feature occurs in the TEP curve for $J_{H} \neq 0$. Note that the TEP for $J_{H}=0.0$ converges monotonically to zero. [25] Transport properties of a metallic system are well described by the semi-classical Boltzmann transport theory [26], which yields the T-linear TEP behavior at low temperature. On the other hand, the TEP for an insulating system is known 27] to be proportional to $1 / T$. Thus it is inferred from the inset in Fig. 1(a) that an insulating phase appears above the dip position temperature $T_{D}$, but disappears with decreasing temperature below $T_{D}$.

As mentioned above, the double exchange model in the dilute carrier concentration limit is illustrated by the concept of the magnetic polaron. [16] Below the peak position
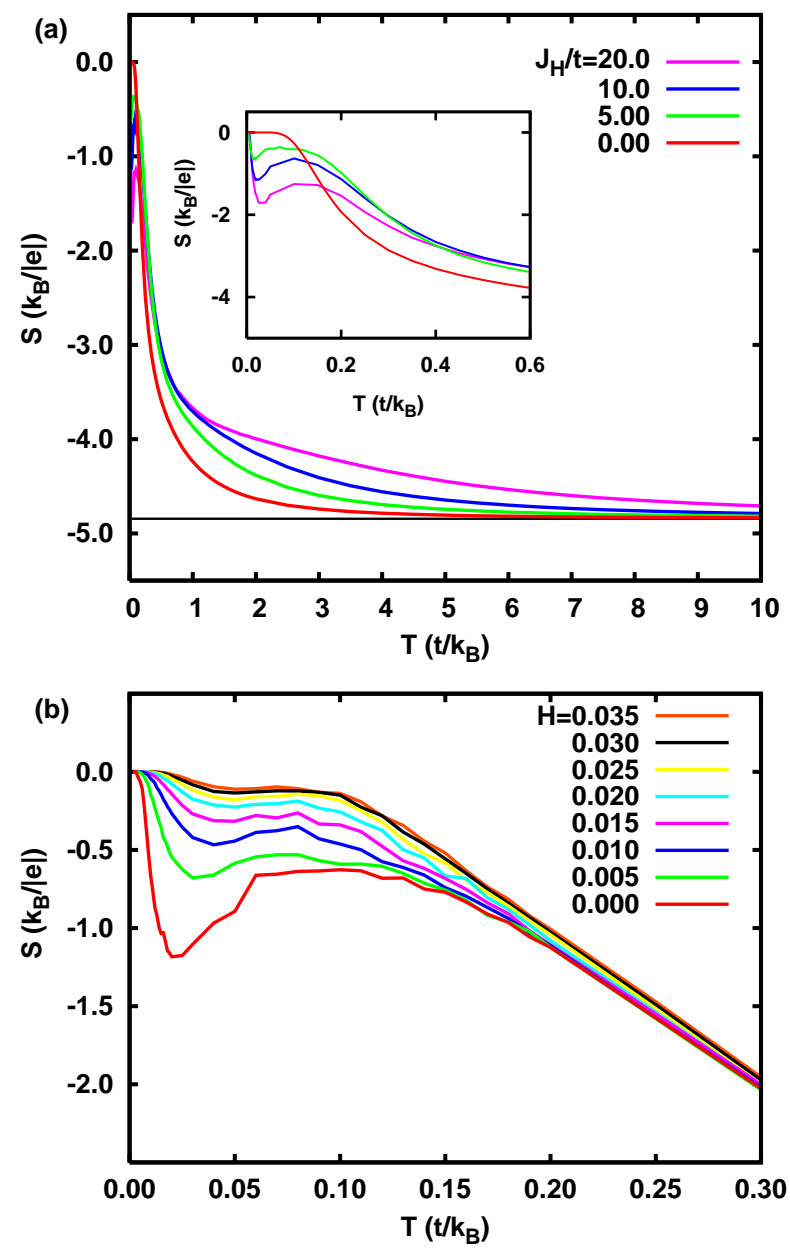

FIG. 1: (color online). (a) The temperature dependence of the thermoelectric power $S(T)$ for different $J_{H}$ parameters in the double exchange model. The thin horizontal solid line which crosses $S=-4.8442 k_{B} /|e|$ represents the value of $S$ calculated by the Heikes formula. The inset in (a) presents the low temperature behaviors of $S(T)$. (b) The magnetic field dependence of $S(T)$ for $J_{H} / t=10.0$. For all cases, $n$ (electron density) is fixed by $n=0.0156$ (one electron in an $8 \times 8$ lattice) .

temperature $T_{P}$, the strong exchange coupling produces the composite quasi-particle of an itinerant electron and local spins, and accordingly the magnetic polaron of FM cluster type is formed. As the clusters are far apart, electrons are trapped and their mobilities are reduced. As the clusters are merged and the FM state begins to appear near $T_{C}$, the mobilities increase again. [16, 28] That is, the insulator to metal transition is caused by the percolation of the magnetic polarons. It is thus evident that the peak and dip feature of the TEP in Fig. 1(a) is closely related to the magnetic polarons which are formed at $T_{P}$ and begin to be percolated at $T_{D}$. 29.

Near $T_{C}$, the TEP exhibits the strong magnetic field dependence as seen in the magnetic and transport properties. [30] As show in Fig. [1(b), the magnitude of the TEP diminishes with increasing the mag- 
netic field and its dip position shifts up in temperature. Also notable is that the magnetothermoelectric power, $\Delta S=S(H)-S(0)$ is positive in this system. This feature is also related to the formation of magnetic polarons. Because the thermal fluctuation of the local spins is suppressed in the presence of the magnetic field, the magnetic polarons are easily formed and merged together. The percolation gives rise to the reduction of the electrical resistivity (the negative magnetoresistance) and the decline of the insulating phase. Then the emerging metallic phase suppresses the peak and dip feature near $T_{C}$ in the TEP curve. Hence the TEP which has a negative value moves up with increasing the magnetic field.

In conclusion, we have investigated the temperature dependence of the TEP for the double exchange model in the dilute carrier concentration limit. The TEP in the high temperature regime follows the Heikes formula independent on $J_{H}$ value. The exchange coupling reduces the TEP in the intermediate temperature regime. Near $T_{C}$, the TEP shows anomalous peak and dip feature and the positive magnetothermoelectric power, which are explained by the concept of the magnetic polaron.

\section{Acknowledgments}

This work was supported by the SRC/ERC program of MOST/KOSEF (R11-2000-071), the basic research program of KOSEF (R01-2006-000-10369-0), and by the POSTECH research fund.
[1] E. L. Nagaev, Phys. Rep. 346, 387 (2001).

[2] H. Ohno, Science 281, 951 (1998).

[3] T. Dietl and H. Ohno, MRS bull. 28, 714 (2003).

[4] C. N. Guy, S. von Molnar, J. Etourneau, and Z. Fisk, Solid State Commun. 33, 1055 (1980).

[5] S. Paschen, D. Pushin, M. Schlatter, P. Vonlanthen, H. R. Ott, D. P. Young, and Z. Fisk, Phys. Rev. B 61, 4174 (2000).

[6] C. Zener, Phys. Rev. 82, 403 (1951).

[7] D. P. Arovas, G. Gómez-Santos, and F. Guinea, Phys. Rev. B 59, 13569 (1999).

[8] B. M. Letfulov and J. K. Freericks, Phys. Rev. B 64, 174409 (2001).

[9] E. Dagotto, T. Hotta, and A. Moreo, Phys. Rep. 344, 1 (2001).

[10] A. Asamitsu, Y. Moritomo, and Y. Tokura, Phys. Rev. B 53, R2952 (1996).

[11] M. F. Hundley and J. J. Neumeier, Phys. Rev. B 55, 11511 (1997).

[12] P. Mandal, Phys. Rev. B 61, 14675 (2000).

[13] N. G. Bebenin, R. I. Zainullina, V. V. Mashkautsan, V. V. Ustinov, and Y. M. Mukovskii, Phys. Rev. B 69, 104434 (2004).

[14] P. G. de Gennes, Phys. Rev. 118, 141 (1960).

[15] N. F. Mott, Contemp. Phys. 31, 373 (1990).

[16] U. Yu and B. I. Min, Phys. Rev. Lett. 94, 117202 (2005).

[17] S. Yunoki, J. Hu, A. L. Malvezzi, A. Moreo, N. Furukawa, and E. Dagotto, Phys. Rev. Lett. 80, 845 (1998).

[18] E. Dagotto, S. Yunoki, A. L. Malvezzi, A. Moreo, J. Hu, S. Capponi, Poilblanc, and N. Furukawa, Phys. Rev. B 58, 6414 (1998).

[19] J. M. Luttinger, Phys. Rev. 136, A1481 (1964).

[20] M. M. Zemljič and P. Prelovšek, Phys. Rev. B 71, 085110 (2005).

[21] B. S. Shastry, Phys. Rev. B 73, 085117 (2006).

[22] P. M. Chaikin and G. Beni, Phys. Rev. B 13, 647 (1976).

[23] N. Metropolis, A. W. Rosenbluth, M. N. Rosenbluth, A. H. Teller, and E. Teller, J. Chem. Phys. 21, 1087 (1953).

[24] See, e.g., W. H. Press, S. A. Teukolsky, W. T. Vetterling, and B. P. Flannery, Numerical recipes in $C$ (Cambridge University Press, New York, 1992).

[25] The unexpected TEP behavior at extremely low temperature for $J_{H}=0$ is thought to be caused by finite size of the system.

[26] N. W. Ashcroft and N. D. Mermin, Solid State Physics (Thomson Learning, Singapore, 1976).

[27] S. M. Girvin, J. Solid State Chem. 25, 65 (1978).

[28] U. Yu and B. I. Min, Phys. Rev. B 74, 094413 (2006).

[29] In our results, $T_{D}$ is only slightly higher than $T_{C}$, and the conductivity variation is maximum near $T_{C}$. But, its magnitude begins to rise up rapidly near $T_{D}$.

[30] B. H. Kim, U. Yu. J. -B. Kim and B. I. Min, J. Appl. Phys. 101, 09G107 (2007). 